\title{
Rapid Evolution of the Ribonuclease A Superfamily: Adaptive Expansion of Independent Gene Clusters in Rats and Mice
}

\author{
Neil A. Singhania, ${ }^{1 *}$ Kimberly D. Dyer, ${ }^{1}$ Jianzhi Zhang, ${ }^{1}$ Madeleine S. Deming, ${ }^{1} * *$ Cynthia A. Bonville, ${ }^{2}$ \\ Joseph B. Domachowske, ${ }^{2}$ Helene F. Rosenberg ${ }^{1}$ \\ ${ }^{1}$ Laboratory of Host Defenses, National Institute of Allergy and Infectious Diseases, National Institutes of Health, Building 10 \\ Bethesda, MD 20892, USA \\ ${ }^{2}$ Department of Pediatrics, State University of New York Health Science Center at Syracuse, Syracuse, NY 13210, USA
}

Received: 8 April 1999 / Accepted: 22 June 1999

\begin{abstract}
The two eosinophil ribonucleases, eosinophil-derived neurotoxin (EDN/RNase 2) and eosinophil cationic protein (ECP/RNase 3), are among the most rapidly evolving coding sequences known among primates. The eight mouse genes identified as orthologs of EDN and ECP form a highly divergent, species-limited cluster. We present here the rat ribonuclease cluster, a group of eight distinct ribonuclease A superfamily genes that are more closely related to one another than they are to their murine counterparts. The existence of independent gene clusters suggests that numerous duplications and diversification events have occurred at these loci recently, sometime after the divergence of these two rodent species ( 10-15 million years ago). Nonsynonymous substitutions per site $\left(d_{\mathrm{N}}\right)$ calculated for the 64 mouse/rat gene pairs indicate that these ribonucleases are incorporating nonsilent mutations at accelerated rates, and comparisons of nonsynonymous to synonymous substitution $\left(d_{\mathrm{N}} / d_{\mathrm{S}}\right)$ suggest that diversity in the mouse ribonuclease cluster is promoted by positive (Darwinian) selection. Although the pressures promoting similar but clearly independent styles of rapid diversification among these primate and rodent genes remain uncertain, our recent
\end{abstract}

\footnotetext{
*Present address: Baylor College of Medicine, Houston, TX

**Present address: New York University School of Medicine, New York, NY

Correspondence to: Helene F. Rosenberg; e-mail: hr2k@nih.gov
}

findings regarding the function of human EDN suggest a role for these ribonucleases in antiviral host defense.

Key words: Ribonucleases - host defense - eosinophils — evolution — rodents

\section{Introduction}

The ribonuclease (RNase) A superfamily is a large group of secretory proteins characterized by shared elements of primary structure and enzymatic activity but (to the extent that they are understood) divergent biological functions (D'Alessio and Riordan 1997; Beintema et al. 1997; Beintema 1998). The elements of shared primary structure include six or eight cysteines in disulfide bonds, which serve to maintain the unique three-dimensional structure of the ribonuclease monomer, and two histidines and one lysine, which form the catalytic site. There are six known human RNase A superfamily ribonucleases, including human pancreatic ribonuclease (RNase 1), the eosinophil ribonucleases (RNases 2 and 3), RNase 4, angiogenin (RNase 5), and RNase k6. Other distinctive family members include bovine seminal ribonuclease (D'Alessio et al. 1997), a dimeric ribonuclease with selective toxicity for malignant cells, and onconase, an amphibian ribonuclease with similar cytotoxic properties (Youle and D'Alessio 1997; Irie et al. 1998).

Our work has focused on the eosinophil ribonucleases, the eosinophil-derived neurotoxin (EDN/RNase 2) 
and eosinophil cationic protein (ECP/RNase 3), which are both major secretory effector proteins of human eosinophilic leukocytes (Rosenberg 1998a). EDN and ECP have emerged as a gene pair relatively recently, sometime after the divergence of the Old World and New World monkeys (Hamann et al. 1990; Rosenberg et al. 1995). We have also found that, since duplication, the genes encoding EDN and ECP have incorporated nonsilent mutations at rates exceeding those of all other functional coding sequences studied among primates, all the while maintaining the eight cysteines, two histidines, and one lysine that are necessary to maintain ribonuclease activity (Rosenberg et al. 1995; Rosenberg \& Dyer 1995). Zhang et al. (1998) have provided evidence in support of positive (Darwinian) selection occurring at the molecular level in the evolution of primate ECP genes. Taken together, the results of these studies suggest that ribonuclease activity must somehow be crucial to one or more physiologic functions of EDN and ECP, a hypothesis borne out by our recent findings regarding the ribonuclease-dependent antiviral activity of human EDN against infection with the single-stranded RNA (ssRNA) respiratory viral pathogen respiratory syncytial virus (RSV) (Domachowske et al. 1998a, 1998b, 1998c).

As part of a larger interest in the evolution and function of the EDN/ECP lineage, we and others have identified the orthologs of these ribonuclease genes in mice. Larson et al. (1996) purified ribonucleases from mouse eosinophils and ultimately identified cDNA sequences of mouse eosinophil-associated ribonuclease (mEAR) -1 and -2 , ribonucleases with $\sim 50 \%$ amino acid sequence identity to human EDN and ECP. Southern analysis suggested the presence of multiple hybridizing species in the mouse genome (Larson et al. 1996); we have since identified six related ribonuclease genes that, together with mEAR-1 and -2 form the mouse ribonuclease (mR) cluster (Batten et al. 1997). These sequences define a unique, species-limited cluster within the RNase A gene family, causing us to remark on the unusual degree of duplication and diversification observed in this specific ribonuclease lineage.

In the work presented here, we intended to explore what we thought would be the nearly identical ribonuclease cluster in rats. In the end, we were unable to identify unique rat orthologs of individual $\mathrm{mR}$ cluster ribonuclease genes; instead, we have found that related but distinct $\mathrm{mR}$ and rat ribonuclease ( $\mathrm{rR}$ ) clusters have evolved independently in rats and mice, with evidence suggesting positive (Darwinian) selection as contributing to the diversification. The numerous duplication and diversification events that generated the rat and nearly all members of the mouse ribonuclease clusters most likely occurred very recently, sometime after the divergence of these two closely related rodent species. This ribonuclease lineage clearly remains the focus of unusual evolutionary pressures both in primates and in rodents; our recent obser- vations regarding the antiviral activity of human EDN have permitted us to speculate as to what these pressures might be.

\section{Materials and Methods}

\section{Genomic DNAs}

Mouse genomic DNA was isolated from cells of the 3 T3 fibroblast cell line derived from the Balb/C mouse strain. Rat genomic DNA (Sprague-Dawley) was purchased from Clontech Laboratories. Human genomic DNA was isolated from peripheral blood leukocytes from normal volunteers.

\section{Isolation of Ribonuclease Coding Sequences by Polymerase Chain Reaction (PCR)}

The PCR reactions were as described previously using rat genomic DNA as template and primers derived from the published coding sequence (Batten et al. 1997; Genbank AF017258); the 5' forward primer, 5'-ATG GGT CCG AAG CTG-3' (encoding amino acids -25 through -21 of the signal sequence; Fig. 1), and the $3^{\prime}$ reverse primer, 5'-AAA TGT CCC ATC CAA-3' (encoding amino acids 127 through 130 at the carboxy terminus). Each PCR reaction included $5 \mu 110 \times$ magnesium-containing reaction buffer (Boehringer Mannheim), $1 \mu \mathrm{l}$ $10 \mathrm{~m} M$ dNTPs, $2.5 \mu$ l of each primer (20 $\mu M$ initial concentration), 1 $\mu l$ template ( $50 \mathrm{ng}$, Clontech), $1 \mu \mathrm{l} \mathrm{Taq}$ polymerase (1 units/ $\mu \mathrm{l}$, Boehringer Mannheim) with $\mathrm{dH}_{2} \mathrm{O}$ to $50 \mu \mathrm{l}$ total volume. The reactions were performed in a Perkin Elmer 9600 thermocycler under the following conditions: $95^{\circ} \mathrm{C}$ for $2 \mathrm{~min}$, followed by 35 cycles of $95^{\circ} \mathrm{C}$ for $20 \mathrm{~s}, 55^{\circ} \mathrm{C}$ for $20 \mathrm{~s}, 72^{\circ} \mathrm{C}$ for $30 \mathrm{~s}$. After the cycling, the reactions were held at $72^{\circ} \mathrm{C}$ for $6 \mathrm{~min}$ and then at $4^{\circ} \mathrm{C}$ until further use. The multiple products present in the resulting amplicons were distinguished by dideoxy sequencing of individual clones isolated on subcloning into the PCR II TA cloning vector (Invitrogen). In an effort to avoid confounding results from potential PCR-induced mutation (estimated to occur under these conditions at roughly 1 in $10^{3}$ to 1 in $10^{4}$ nucleotides amplified), each sequence reported was isolated at least three times from independent cycling reactions. In addition, only sequences with three or more changes in amino acid sequence were considered in this analysis.

\section{Southern Analysis}

Rat and human genomic DNAs $(20 \mu \mathrm{g})$ were restriction-digested with Pst I prior to gel electrophoresis and transfer by standard methods. Blots were prehybridized prior to overnight hybridization at $60^{\circ} \mathrm{C}$ in buffer containing $50 \%$ formamide, 6X SSPE, $10 \%$ dextran sulfate, $0.5 \%$ sodium dodecyl sulfate, $50 \mu \mathrm{g} / \mathrm{ml}$ sheared salmon sperm DNA with ${ }^{32} \mathrm{P}$-labeled random-primed probe including the complete coding sequence of rat ribonuclease $\mathrm{rR}-12$ at $4 \times 10^{6} \mathrm{cpm} / \mathrm{ml}$. Blots were washed at $60^{\circ} \mathrm{C}$ in $0.2 \mathrm{X} \mathrm{SSPE}$ and evaluated by autoradiography.

\section{Northern Analysis}

A rat multitissue Northern blot was purchased from Clontech Laboratories and prehybridized prior to sequential hybridization in buffer and with conditions as described above with ${ }^{32} \mathrm{P}$-labeled complete coding sequences of rR-1, rR-5, and rR-12. Blots were washed as described above and evaluated by autoradiography. The integrity and relative 

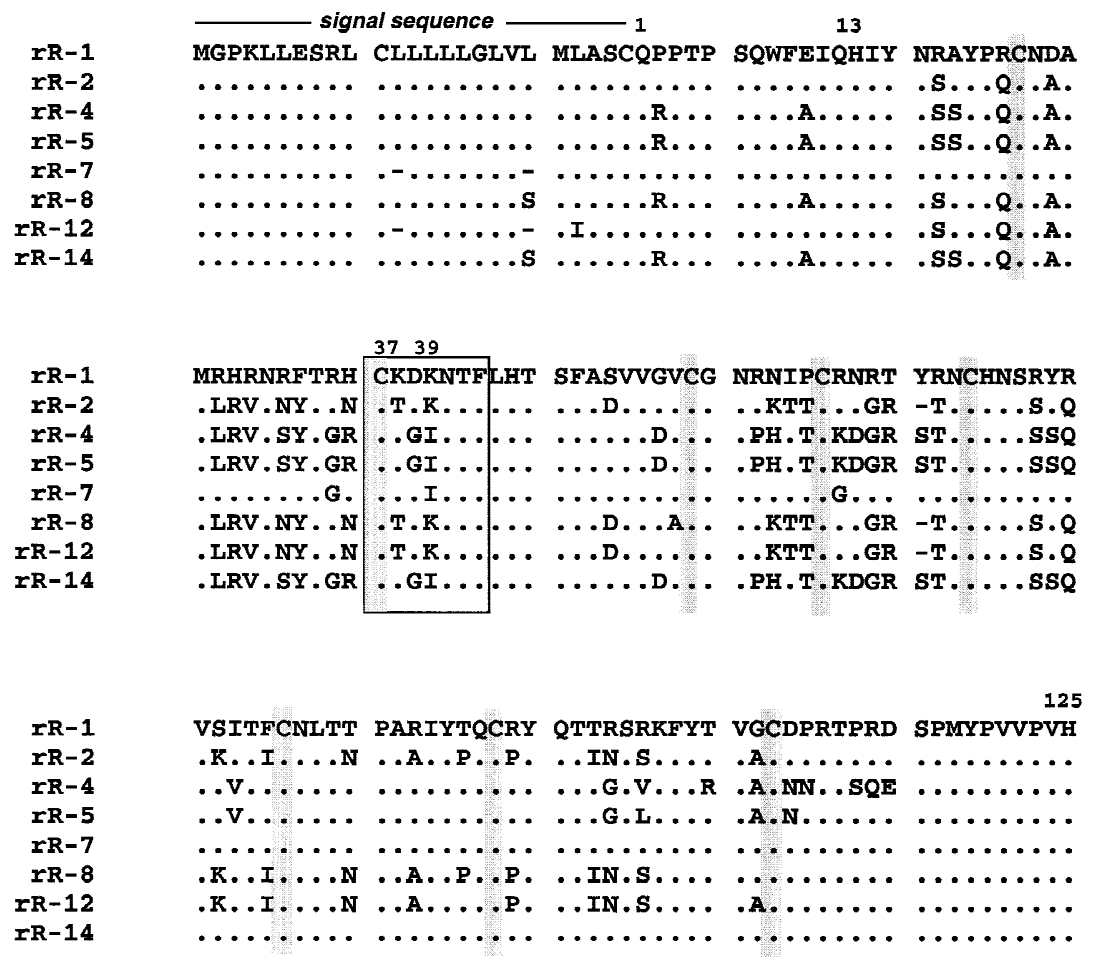

\begin{tabular}{|c|c|c|c|c|}
\hline & & length & isoelectric pt. & \#ArQ \\
\hline rR-1 & LDGTF & 130 & 10.9 & 18 \\
\hline rR-2 & $\ldots \ldots$ & 129 & 8.4 & 7 \\
\hline rR-4 & $\cdots \cdots$ & 130 & 8.9 & 8 \\
\hline rR-5 & $\cdots \cdots$ & 130 & 8.9 & 8 \\
\hline rR-7 & $\ldots \ldots$ & 130 & 10.5 & 16 \\
\hline rR-8 & $\ldots \ldots$ & 129 & 8.9 & 8 \\
\hline rR-12 & $\ldots \ldots$ & 129 & 8.4 & 7 \\
\hline$r R-14$ & $\ldots \ldots$ & 130 & 9.2 & 10 \\
\hline
\end{tabular}

Fig. 1. Alignment of the amino acid sequences encoded by rat $r R$ ribonuclease cluster genes. Dots indicate sequence conservation; disparity from the sequence of $\mathrm{rR}-1$ is indicated by single letter amino acid sequence code. Features typical of the RNase A gene superfamily include the consensus amino-terminal signal sequence, eight cysteines (shaded), and catalytic histidines $\left(\mathrm{H}^{13}\right.$ and $\left.\mathrm{H}^{125}\right)$ and lysine $\left(\mathrm{K}^{37}\right)$ within a conserved motif (box); the lysine in ribonucleases $\mathrm{rR}-2, \mathrm{rR}-8$,

loading of the DNA and RNA of the Southern and Northern blots, respectively, were determined by probing with a radio labeled betaactin probe.

\section{Recombinant Proteins}

Recombinant $\mathrm{rR}-5$ and $\mathrm{rR}-12$ proteins were prepared in the pF-CTS bacterial expression system (Eastman Kodak) as described previously (Rosenberg and Dyer 1995, 1997). After induction with $1 \mu M$ IPTG (Rosenberg 1998b), bacteria were harvested, and whole cell lysates were dialyzed overnight against $50 \mathrm{~m} M$ tris ( $\mathrm{pH}$ 8.0) and $1 \mathrm{mM} \mathrm{NaCl}$. Recombinant protein was isolated to homogeneity by sequential heparin sepharose chromatography ( $1 \mathrm{~m} M$ to $2 M$ salt gradient) followed by size selection on Superdex 75 in a $50 \mathrm{~m} M$ tris (pH 8.0) and $150 \mathrm{mM}$ $\mathrm{NaCl}$ mobile phase. Final concentration was determined by BCA assay and by quantitative Western blotting (Rosenberg and Dyer 1995, 1997).

\section{Ribonuclease Assay}

Ribonuclease activity was measured as the generation of acid-soluble ribonucleotides from acid-insoluble yeast tRNA substrate per unit time

and $\mathrm{rR}-12$ is in a shifted position $\left(\mathrm{K}^{39}\right)$. Genbank accession numbers for these sequences are: rR-1, AF171641; rR-2, AF171642; rR-4, AF171643; rR-5, AF171644; rR-7, AF171645; rR-8, AF171646; rR12, AF171647; rR-14, AF171648. Primer encoded sequence includes amino acids -21 to -25 of the signal sequence and 127 to 130 at the carboxy terminus. Length was calculated beginning at $\mathrm{Q}^{1}$.

as described previously (Rosenberg and Dyer 1995, 1997). Time points were measured in triplicate, and rates were determined as the best-fit line through zero and four successive time points as per the SLOPE algorithm of Microsoft Excel.

\section{Evolutionary and Statistical Analysis}

Evolutionary analysis was performed with the assistance of the MEGA analysis program (Kumar et al. 1993; Zhang et al. 1998) and the GAP algorithm of the Wisconsin Genetics Computer Group program available at the National Institutes of Health. Primer-encoded sequence was not included in any of these analyses.

\section{Results}

\section{Identification of Novel Rat Ribonuclease Genes}

An alignment of the amino acid sequences encoded by the eight rat ribonuclease genes is shown in Fig. 1. Each 
sequence has features typical of members of the RNase A superfamily. Each sequence includes an amino terminal signal peptide, with the amino terminus of the processed form of the protein indicated as glutamine- $1\left(\mathrm{Q}^{1}\right)$; glutamine-1 was found at the amino termini of the related ribonucleases mEAR- 1 and -2 by direct sequencing of purified proteins (Larson et al. 1996). The processed protein sequences include either 129 or 130 amino acids, each with the appropriately spaced cysteines (shaded) typical of RNase A family ribonucleases as well as the two histidines $\left(\mathrm{H}^{13}\right.$ and $\left.\mathrm{H}^{125}\right)$ analogous to those found in the catalytic site of RNase A. The calculated isoelectric points of all encoded proteins range from 8.4 to 9.2, except for $\mathrm{rR}-1$ and $\mathrm{rR}-7$, which are cationic ribonucleases with isoelectric points exceeding 10. Rat rR-7 differs from "rat ECP" identified by Nittoh et al. (1997) by a single amino acid, and they are thus considered identical by this analysis (see Materials and Methods).

Overall, the most conserved areas of amino acid sequence are found near the amino and carboxy termini; most of the heterogeneity can be found in the region just preceding the conserved CKXXNTF motif (amino acids 27-35) and in a second region just distal (amino acids 56-67). Interestingly, the CKXXNTF motif, which is invariant in all RNase A ribonucleases identified to date and contains the catalytic lysine $\left(\mathrm{K}^{37}\right)$, has been altered in three of these rat ribonucleases. In ribonucleases $\mathrm{rR}-2$, rR-8, and rR-12, the catalytic lysine is shifted two positions downstream to become $\mathrm{K}^{39}$. The cationic ribonuclease rR-1 has two lysines, one shifted and one at the traditional locale. The three remaining ribonucleasesrR-4, rR-5, and rR-14-have no unusual features. All three of these "traditional" ribonucleases include regions of sequence that match the tryptic peptides of ribonuclease RL2 recently reported by Zhao et al. (1998).

Restriction-digested rat and human genomic DNA were probed with the coding sequence of rR-12 (Fig. 2), which has $>80 \%$ nucleotide sequence identity with all other rat $\mathrm{rR}$ cluster ribonucleases identified in this study. Multiple hybridizing sequences were detected in rat genomic DNA, providing support for the genomic basis of the cluster as described. No sequences were detected in the lanes containing human DNA. The integrity of the rat and human DNA was confirmed by hybridization with a beta-actin probe (data not shown).

\section{Relationship to Human RNase A Superfamily Ribonucleases}

Shown in Fig. 3 are nucleotide sequence identities calculated for individual $\mathrm{rR}$ cluster ribonucleases and each of the six human RNase A family ribonucleases. In contrast to the less specific results obtained in our analysis of the mouse ribonuclease cluster (Batten et al. 1997), these results demonstrate that the $\mathrm{rR}$ cluster ribonucleases are clearly more closely related to EDN/RNase 2 and ECP/

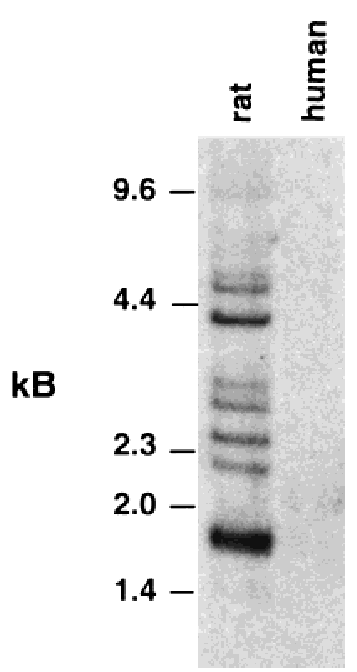

Fig. 2. Genomic Southern analysis. Genomic DNAs isolated from rat, and human tissue were restriction-digested with Pst-I and probed with a ${ }^{32} \mathrm{P}$-labeled coding sequence of rR-12 (> 80\% nucleotide sequence identity with all $\mathrm{rR}$ ribonucleases identified). The integrity of the genomic DNA was confirmed by hybridization with a beta-actin cDNA probe (data not shown).

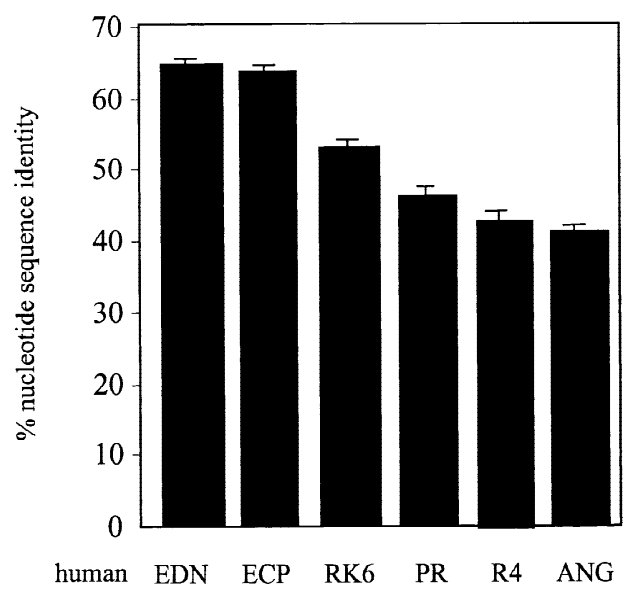

Fig. 3. Human RNase A superfamily and $r R$ cluster ribonucleases. Nucleotide sequence identity between individual human and rat $\mathrm{rR}$ ribonucleases was determined via the GAP algorithm of WGCG; each bar represents the average of eight rat/human ribonuclease pairs \pm standard deviation. Genbank accession numbers for the human sequences are: M24157, EDN (eosinophil-derived neurotoxin)/RNase 2; X15161, ECP (eosinophil cationic protein)/RNase 3; U64998, RK6/ RNase 6; X79235, PR (pancreatic ribonuclease)/RNase 1; U36775, R4/RNase 4; M11567, ANG (angiogenin)/RNase 5.

RNase 3 than they are to any of the other human members of this superfamily. Although we cannot completely rule out the possibility that another, more closely related human ribonuclease exists, current evidence suggests that these rat ribonuclease genes are the orthologs of human EDN and human ECP.

\section{Ribonuclease Activity}

The ribonuclease activity of the recombinant form of the traditional ribonuclease $\mathrm{rR}-5$ was compared to that of the 
Table 1. Enzymatic activity of rat ribonucleases rR-5 and rR-12

\begin{tabular}{llc}
\hline RNase & $\mathrm{R}^{\mathrm{a}}(\mathrm{mU} / \mathrm{ul})$ & Activity $(\mathrm{pmol} / \mathrm{m})$ \\
\hline- & - & 0.47 \\
rR-5 & - & 18.3 \\
rR-5 & 1 & 14.9 \\
rR-5 & 5 & 8.5 \\
rR-12 & - & 0.46 \\
\hline
\end{tabular}

Ribonuclease activities (pmol acid-soluble ribonucleotides generated per min from acid-insoluble yeast tRNA) of recombinant proteins rR-5 $(0.3 \mathrm{pmol} / \mathrm{ml})$ and $\mathrm{rR}-12(4 \mathrm{pmol} / \mathrm{ml})$ were determined as described (Rosenberg and Dyer 1995, 1997). Rates were determined from the slope of the best-fit line drawn through measured zero and four successive time points, each measured in triplicate.

${ }^{\mathrm{a}} \mathrm{RI}=$ placental ribonuclease inhibitor.

shifted-lysine ribonuclease $\mathrm{rR}-12$ (Table 1). As is typical for members of the RNase A superfamily, rR-5 degrades the yeast tRNA substrate, and its activity can be inhibited in a dose-dependent fashion by placental ribonuclease inhibitor (RI). Recombinant rR-12 has no activity detectable above background level.

\section{Evolutionary Dendrogram}

The relationships among the mouse $\mathrm{mR}$ and rat $\mathrm{rR}$ ribonucleases are shown in the dendrogram in Fig. 4. This dendrogram displays a number of interesting features. First is the observation that these rat ribonucleases form subclusters, with traditional (rR-4, -5 , and -14 ), shiftedlysine (rR-2, -8, and -12), and cationic (rR-1 and -7) ribonucleases in distinct subgroups based on nucleotide sequence homology. Second, the rat $\mathrm{rR}$ and the mouse $\mathrm{rR}$ ribonucleases are clearly more closely related to one another within each species, with no evidence for any 1:1 interspecies pairing. It is not clear from these data whether the $\mathrm{rR}$ cluster diverged from the $\mathrm{mR}$ cluster in its entirety, or whether certain members of the $\mathrm{mR}$ cluster, specifically mR-4 and mR-5, represent the direct descendants of more ancient sequences. Although the tree presented is most consistent with the data, the statistical support for placing these two sequences directly within the cluster is relatively weak (bootstrap value $=58$ ). Thus, it remains possible that the $\mathrm{rR}$ and the six other members of the $\mathrm{mR}$ cluster are descendants of a third ribonuclease that was not a common ancestor of mR-4 or mR-5. Thus, most conservatively, the results shown here suggest that the duplication and diversification events generating the rat and six of the eight members of the mouse ribonucleases occurred independently, after the divergence of these two closely related rodent species, a date estimated at 10-15 million years ago.

\section{Synonymous and Nonsynonymous Substitution}

We have calculated synonymous $\left(d_{\mathrm{S}}\right)$ and nonsynonymous $\left(d_{\mathrm{N}}\right)$ substitution per respective site for human

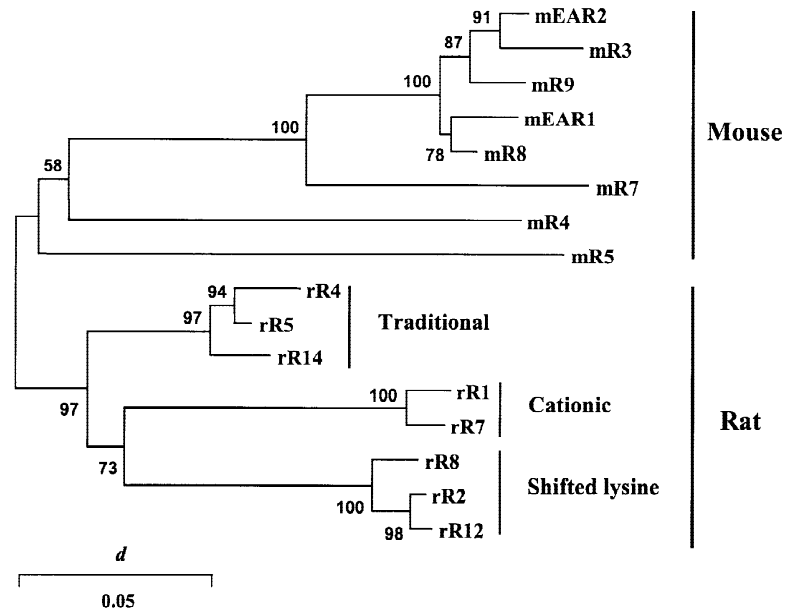

Fig. 4. Evolutionary dendrogram depicting the relationships among the mouse $\mathrm{mR}$ and rat $\mathrm{rR}$ cluster ribonucleases. The tree was constructed using the neighbor joining method with Kimura's distances (Kimura 1980). The tree is rooted on the interior branch between the mouse $\mathrm{mR}$ and rat $\mathrm{rR}$ ribonuclease gene clusters using the sequences of human EDN and ECP as outgroups. Values at the interior nodes are bootstrap percentages derived from 500 replications (Kumar et al. 1993). Genbank accession numbers include mR-7, AF171649; mR-8, AF171650; mR-9, AF171651.

EDN and rat $r R$ ribonucleases (eight pairs), human EDN and mouse $\mathrm{mR}$ ribonucleases (eight pairs) and mouse $\mathrm{mR}$ and rat $\mathrm{rR}$ ribonucleases (64 pairs), and compared these distances to those calculated by Makalowski and Boguski (1998) (Fig. 5). Whereas the values calculated for $d_{\mathrm{N}}$ and $d_{\mathrm{S}}$ for human/mouse and human/rat ribonuclease pairs are substantially greater than the mean values calculated for these interspecies pairings, they do not exceed the upper limits observed for other, rapidly evolving coding sequences. Similarly, the ratios of $d_{\mathrm{N}} / d_{\mathrm{S}}$ are unremarkable for the human/rodent ribonuclease pairs. In contrast, the comparisons between mouse and rat ribonucleases are quite remarkable. The values for $d_{\mathrm{S}}$ fall well within the range calculated for 470 mouse/rat orthologs, consistent with the identification of the $\mathrm{mR}$ and rR clusters as interspecies orthologs, but the values calculated for $d_{\mathrm{N}}$ fall at or near the highest values in this range, suggesting that these ribonuclease gene clusters are incorporating nonsilent mutations at rates exceeding those of most other coding sequences known in these two rodent species. A more complete analysis of the evolution of these rat and mouse clusters is shown in Fig. 6 in which we compare $d_{\mathrm{N}}$ and $d_{\mathrm{S}}$ for all pairs of rodent ribonucleases (mouse/mouse, rat/rat, and mouse/rat). These data provide strong support for positive (Darwinian) selection as a contributing factor to the diversification observed among the mouse ribonuclease genes, as $d_{\mathrm{N}} / d_{\mathrm{S}}$ exceeds unity in all 27 mouse/mouse pairs. Using the dendrogram shown in Figure 4, all branches linking $\mathrm{mR}$ cluster genes were evaluated as described by Zhang et al. (1998). For this set of branches, the total $d_{\mathrm{N}}$ was calculated at $0.586 \pm 0.051$, and total $d_{\mathrm{S}}, 0.353 \pm 0.061$, with $d_{\mathrm{N}}>d_{\mathrm{S}}(p=0.002)$ providing overwhelming sup- 


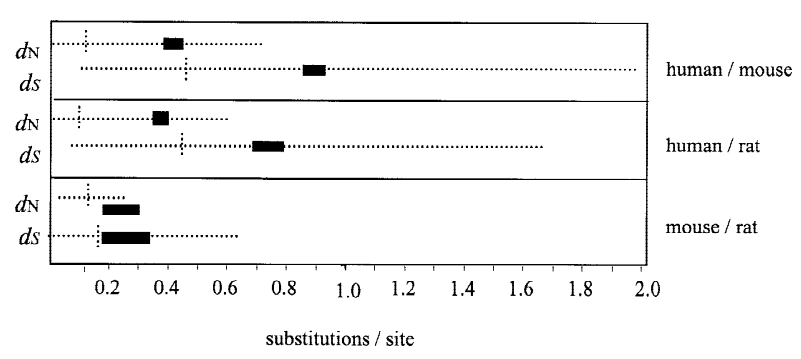

Fig. 5. Nonsynonymous $\left(d_{\mathrm{N}}\right)$ and synonymous $\left(d_{\mathrm{S}}\right)$ substitution: human, mouse, and rat ribonucleases. Pairwise distances were calculated for human EDN/mouse mR cluster (eight pairs), human EDN/rat rR cluster (eight pairs), and mouse $\mathrm{mR} / \mathrm{rat} \mathrm{rR}$ cluster ribonucleases (64 pairs); range of values calculated is indicated by the horizontal black bars. Dotted lines indicate the range and weighted means calculated for 1,138 mouse/human, 1,212 rat/human, and 470 mouse/rat coding sequence pairs by Makalowski and Boguski (1998).

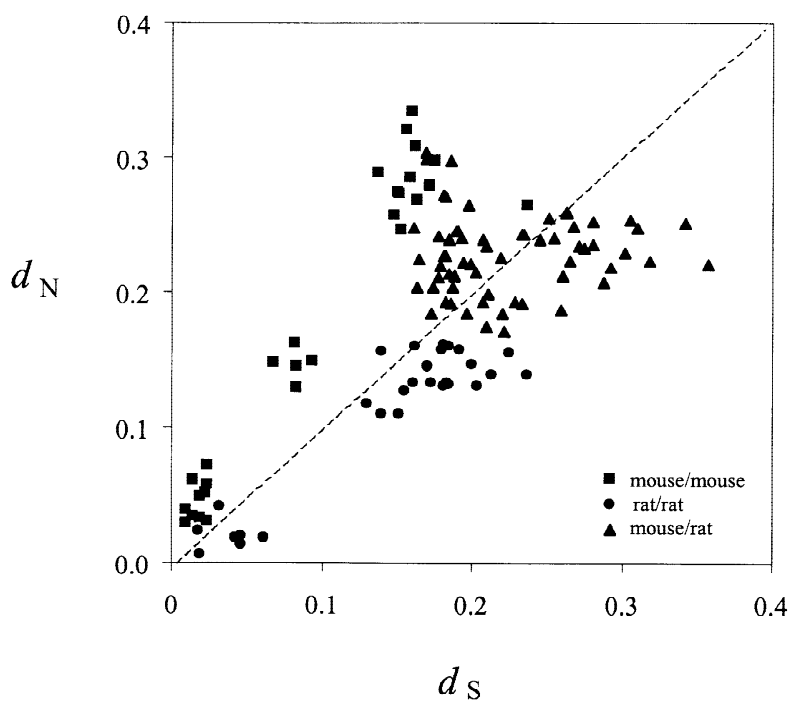

Fig. 6. Nonsynonymous $\left(d_{\mathrm{N}}\right)$ and synonymous $\left(d_{\mathrm{S}}\right)$ substitution: mouse and rat ribonucleases. Pairwise distances were calculated using the method of Zhang et al. (1998), with the transition/transversion ratio estimated at 0.85 using Kimura's method (Kimura 1980). Values shown include intraspecies (squares, mouse/mouse; circles, rat/rat) and interspecies (triangles, mouse/rat) comparisons.

port for a role for positive selection in the diversification of the mouse $\mathrm{mR}$ ribonuclease gene cluster.

\section{Expression of Rat Ribonuclease mRNA}

Shown in Fig. 7 is a rat multitissue Northern blot probed with the coding sequences of $\mathrm{rR}-1, \mathrm{rR}-5$, and $\mathrm{rR}-12$. Although we cannot know for certain exactly how many of the $\mathrm{rR}$ cluster ribonucleases are represented in any one of the three bands shown, the data indicate that $\mathrm{rR}$ ribonucleases are expressed in rat lung. The significance of this finding to the proposed function of these genes is considered in the Discussion.

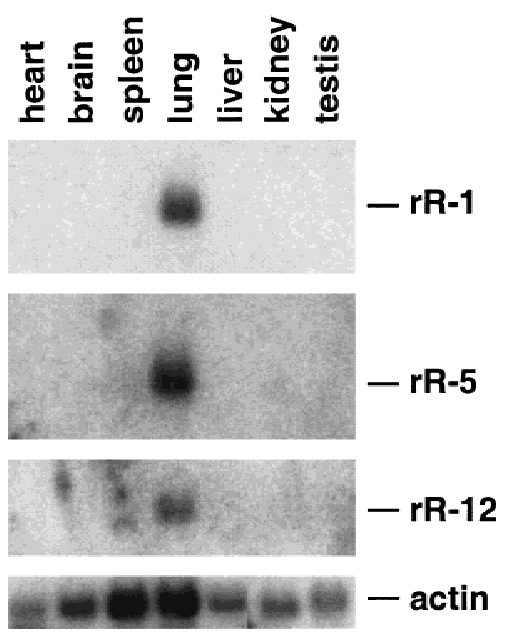

Fig. 7. Tissue-specific expression of rat $r R$ ribonucleases. Probes include the ${ }^{32} \mathrm{P}-$ labeled coding sequences of rR-1 (cationic), rR-5 (traditional), and rR-12 (shifted lysine) ribonucleases. Blots were also probed with a beta-actin cDNA probe to demonstrate relative loading of each lane.

\section{Discussion}

In this work we have identified and characterized genes of a novel rat (rR) ribonuclease cluster. The cluster ribonucleases are members of the RNase A superfamily and are, to the best of our knowledge, orthologs of the EDN/ECP ribonuclease lineage described in primates (Rosenberg et al. 1995). Despite the homology of each of these rat ribonucleases to members of the previously identified mouse ribonuclease $(\mathrm{mR})$ gene cluster (Larson et al. 1996; Batten et al. 1997), the rat ribonucleases form their own (rR) cluster, as each is more closely related to another $\mathrm{rR}$ ribonuclease than it is to any one of the known $\mathrm{mR}$ ribonucleases shown. These results suggest that the $\mathrm{rR}$ and at least six of the eight $\mathrm{mR}$ ribonucleases (see Results) have evolved independently and that multiple duplication and diversification events occurred after the divergence of these two closely related rodent species. Although the dendrogram as presented infers that distinct gene duplication and diversification events occurred after the divergence of rats and mice, it remains possible that gene conversion has played a role in homogenizing previously distinct RNase A superfamily genes within each species. We believe this interpretation is less likely, as there remain substantial differences between ribonucleases within each cluster, most notably for the mouse $\mathrm{mR}$ cluster, as is clear from the extent of nonsynonymous substitutions per site among mouse/ mouse pairs as described in Fig. 6. Furthermore, synonymous substitutions for mouse/mouse and rat/rat pairs are not clustered, but scattered throughout the length of the sequences. Finally, among the rat sequences, novel features have emerged, such as the aforementioned shifted lysine within the otherwise invariant motif, that suggest diversification as opposed to homogenization after gene duplication. Overall, we were intrigued to find that the 
recent, rapid-paced evolution described for EDN/ECP genes of primates is also occurring, albeit in an independent and somewhat different fashion, in rodents.

Several of these $\mathrm{rR}$ cluster ribonucleases have unusual features worthy of comment. Perhaps the most intriguing are $\mathrm{rR}-2, \mathrm{rR}-8$, and $\mathrm{rR}-12$, in which there is a clear disruption in the otherwise invariant RNase A superfamily motif-here, $\mathrm{CK}^{37} \mathrm{XXNTF}$ - surrounding the catalytic lysine. In three $\mathrm{rR}$ cluster ribonucleases, the lysine $\left(\mathrm{K}^{37}\right)$ is shifted two positions downstream (to $\mathrm{K}^{39}$ ), converting the motif to $\mathrm{CXXK}^{39} \mathrm{NTF}$. As might be expected, rR-12 had no detectable catalytic activity against the standard yeast tRNA substrates. As we have no direct information on their transcription and/or translation, we cannot rule out the possibility that $\mathrm{rR}-2,-8$, and -12 are functionally pseudogenes. At the moment, the biologic function (potential or otherwise) of these three "shifted-lysine" rR ribonucleases remains unknown.

Two of the rR cluster ribonucleases, $r R-1$ and $r R-7$, are significantly more cationic than the others. Although rR-1 and rR-7 are nearly as cationic as hECP, sequence comparisons indicate that they are no more closely related to hECP overall than they are any of the other, noncationic rat ribonucleases. While the pressures to which these genes are responding remain unclear, there appears to be a need for cationic ribonucleases both in primates and in rodents.

Taken together, we have found that both rodent and primate ribonucleases are incorporating nonsilent mutations at a rapid rate and that each species has generated arginine-enriched cationic ribonucleases. These findings suggest that the primate and the rodent ribonucleases of the EDN/ECP lineage are responding to what may be independent but related evolutionary constraints.

Finally, we were intrigued to find that mRNAs encoding $\mathrm{rR}$ ribonucleases are expressed in rat lung (Fig. 7). Although we cannot know for certain how many different cluster ribonucleases are expressed in this tissue, the lung is an interesting location for $\mathrm{rR}$ ribonucleases in general, given our recent findings regarding the function of the human ortholog, hEDN. Until recently, hEDN's physiologic function was a complete mystery; its name was derived from its nonphysiologic toxicity to rabbit Purkinje cells (Durack et al. 1979, 1981). As part of our ongoing quest to determine a physiologic, ribonucleasedependent function for this protein, we have begun to explore the relationships linking human EDN's primary host cell-the human eosinophilic leukocyte-to diseases caused by the ssRNA respiratory viral pathogen RSV (Garofalo et al. 1992; Kimpen et al. 1992, 1996; Harrison et al. 1999). To this end, we have recently shown that recombinant human EDN mediates the dosedependent, ribonuclease-dependent reduction in infectivity of RSV in assays performed in vitro (Domachowske et al. 1998a, 1998b), an antiviral effect that involves a specific, saturable interaction between EDN and an asyet-unidentified target (Domachowske et al. 1998c).

Based on these findings, we have hypothesized that the rapid evolution of the primate EDN genes may be in response to pressures promoting antiviral host defense against specific primate ssRNA viral pathogens, such as (but not limited to) RSV. Given that the mouse and rat ribonucleases are undergoing similar but independent styles of rapid evolution, we are intrigued by the possibility that this ribonuclease lineage may be responding to similar but independent evolutionary pressures in rodent species, pressures promoting host defense against specific rodent ssRNA viral pathogens.

Our hypothesis-that these ribonucleases are responding to similar but independent pressures both in primates and in rodents, and that the EDN/ECP ribonucleases are an as-yet-unexplored arm of innate and specific antiviral host defense-awaits formal testing in vivo in relevant animal model systems.

Acknowledgments. We would like to thank Dr. Harry L. Malech, Dr. John I. Gallin, and Dr. Leonard B. Weiner for their ongoing support of the work in progress in our laboratories. JBD is a fellow of the American Lung Association.

\section{References}

Batten D, Dyer KD, Domachowske JB, Rosenberg HF (1997) Molecular cloning of four novel murine ribonuclease genes: unusual expansion within the ribonuclease A gene family. Nucl Acids Res 25:4235-4239

Beintema JJ (1998) The ribonuclease A superfamily. Cell Mol Life Sci 54:763-765

Beintema JJ, Breukelman HJ, Carsana A, Furia A (1997) Evolution of vertebrate ribonucleases: ribonuclease A superfamily. In: D'Alessio G, Riordan JF (eds) Ribonucleases, structures and functions. Academic Press, San Diego, CA, pp 245-269

D'Alessio G, Riordan, JF (1997) Ribonucleases, structures and functions. Academic Press, San Diego, CA

D’Alessio G, Di Donato A, Mazzarella L, Piccoli R (1997) Seminal ribonuclease: the importance of diversity. In: D'Alessio G, Riordan JF (eds) Ribonucleases, structures and functions. Academic Press, San Diego, CA, pp 383-425

Domachowske JB, Dyer KD, Bonville CA, Rosenberg HF (1998a) Recombinant human eosinophil-derived neurotoxin/RNase 2 functions as an effective antiviral agent against respiratory syncytial virus. J Infect Dis 177:1458-1464

Domachowske JB, Dyer KD, Adams AG, Leto TL, Rosenberg, HF (1998b) Eosinophil cationic protein (ECP/RNase 3): another eosinophil ribonuclease with direct antiviral activity. Nucl Acids Res 26:3358-3363

Domachowske JB, Bonville CA, Dyer KD, Rosenberg HF (1998c) Evolution of antiviral activity in the ribonuclease A gene superfamily: evidence for a specific interaction between eosinophilderived neurotoxin (EDN/RNase 2) and respiratory syncytial virus. Nucl Acids Res 26:5327-5332

Durack DT, Sumi SM, Klebanoff SJ (1979) Neurotoxicity of human eosinophils. Proc Natl Acad Sci USA 76:1443-1447

Durack DT, Ackerman SJ, Loegering DA, Gleich GJ (1981) Purification of human eosinophil-derived neurotoxin. Proc Natl Acad Sci USA 78:5165-5169 
Garofalo R, Kimpen JL, Welliver RC, Ogra PL (1992) Eosinophil degranulation in the respiratory tract during naturally acquired respiratory syncytial virus infection. J Pediatr 120:28-32

Hamman KJ, Ten RM, Loegering DA, Jenkins RB, Heise MT, Schad CR, Pease LR, Gleich GJ, Barker RL (1990) Structure and chromosomal localization of the human eosinophil-derived neurotoxin and eosinophil cationic protein genes: evidence for intronless coding sequences in the ribonuclease gene superfamily. Genomics 7 : 535-546

Harrison AM, Bonville CA, Rosenberg HF, Domachowske JB (1999) Respiratory syncytial virus infection induces expression of macrophage inflammatory protein 1-alpha (MIP-1alpha) in the human lower respiratory tract in vivo: association with eosinophil recruitment and degranulation. Am J Resp Dis Crit Care Med 159:19181924

Irie M, Nitta K, Nonaka T (1998) The biochemistry of frog ribonucleases. Cell Mol Life Sci 54:775-784

Kimpen JL, Garofalo R, Welliver RC, Fujihara K, Ogra PL (1992) Activation of human eosinophils by respiratory syncytial virus. Pediatric Res 32:160-164

Kimpen JL, Garofalo R, Welliver RC, Fujihara K, Ogra PL (1996) An ultrastructural study of the interaction of human eosinophils with respiratory syncytial virus. Pediatr Allergy Immunol 7:48-53

Kimura M (1980) A simple method for estimating evolutionary rate of base substitution through comparative studies of nucleotide sequences. J Mol Evol 16:111-120

Kumar S, Tamura K, Nei M (1993) Molecular evolutionary genetics analysis, version 1.02. Institute of Molecular Evolutionary Genetics, Pennsylvania State University, State College, PA

Larson KA, Olson EA, Madden BJ, Gleich GJ, Lee NA, Lee JJ (1996) Two highly homologous ribonuclease genes expressed in mouse eosinophils identify a larger subgroup of the mammalian ribonuclease superfamily. Proc Natl Acad Sci USA 93:12370-12375
Makalowski W, Boguski MS (1998) Evolutionary parameters of the transcribed mammalian genome: an analysis of 2,820 orthologous rodent and human sequences. Proc Natl Acad Sci USA 95:94079413

Nittoh T, Hirakata M, Mue S, Ohuchi K (1997) Identification of a cDNA encoding rat eosinophil cationic protein/eosinophilassociated ribonuclease. Biochim Biophys Acta 1351:42-46

Rosenberg HF (1998a) The eosinophil ribonucleases. Cell Mol Life Sci 54:795-803

Rosenberg HF (1998b) Isolation of recombinant secretory proteins by limited induction and quantitative harvest. Biotechniques 24:188192

Rosenberg HF, Dyer KD (1995) Eosinophil cationic protein and eosinophil-derived neurotoxin: evolution of novel function in a primate ribonuclease gene family. J Biol Chem 270:21539-21544

Rosenberg HF, Dyer KD (1997) Diversity among the primate eosinophil-derived neurotoxin genes: a specific carboxy-terminal sequence is necessary for enhanced ribonuclease activity. Nucl Acids Res 35:3532-3536

Rosenberg HF, Dyer KD, Tiffany HL, Gonzalez M (1995) Rapid evolution of a unique family of primate ribonuclease genes. Nature Gen 10:219-223

Youle RJ, D’Alessio G (1997) Anti-tumor RNases. In: D'Alessio G, Riordan JF (eds) Ribonucleases, structures and functions. Academic Press, San Diego, CA, pp 491-514

Zhang J, Rosenberg HF, Nei M (1998) Positive Darwinian selection after gene duplication in primate ribonuclease genes. Proc Natl Acad Sci USA 95:3708-3713

Zhao W, Kote-Jarai Z, van Santen Y, Hofsteenge J, Beintema JJ (1998) Ribonucleases from rat and bovine liver: purification, specificity and structural characterization. Biochim Biophys Acta 1384:55-65 